\title{
A Rationale for Multi-modality in Multimedia Instructional Design
}

\author{
Sanja Kišiček, Tomislava Lauc \\ Faculty of Humanities and Social Sciences, University of Zagreb \\ Ivana Lucica 3, 10000 Zagreb, Croatia \\ \{smatic, tlauc\}@ffzg.hr
}

\section{Summary}

This paper describes a multi-modal approach to multimedia instructional design in a virtual learning environment. The research was conducted within an online course offering multimedia and multi-modal lessons, each of which was comprised of three different types of resources, specifically designed for the acquisition of computer literacy skills. The resources vary from textual resources, pictorial resources accompanied by text and video resources. All the resources are designed by the course lecturers, i.e. the authors of this paper. The purpose of the research was to investigate user perceptual modalities in terms of their preference towards educational multimedia for the acquisition of computer literacy. The methodology of this research is based on data mining techniques through log data which represents user navigation and behavior within the learning management system. The approach fosters self-regulated technology enhanced learning where the end user has control over the choice of learning resources according to personal preferences.

The results of cluster analysis show that there are consistent user preferences in selecting a particular type of resources representing particular modality. The results provide a rationale for a multi-modal approach in designing a learning system based on multimedia instructional design and developed specifically for the acquisition of computer literacy, as a requirement for information literacy of the 21 st century.

Keywords: virtual learning environment, multimedia instructional design, perceptual modalities, user preferences, multi-modality

\section{Introduction}

Courses supported by learning management systems (LMS) manifest various contexts, activities and resources for learners. In the field of computer literacy, a great amount of learning content is accessible in a variety of multimedia formats, with the resources ranging from text over image to video. In 1955, Jakob Nielsen anticipated that by 2010 , video will be the major multimedia format for information presentation on a personal computer (Nielsen, 1955). He also anticipated that today there would be large amounts of hypertext throughout uni- 
versities in the form of shared virtual information. Was Nielsen right? The amount of massive open online courses (MOOCs) today is a witness that he was. Technology Enhanced Learning attracts great interest, promising the possibility of individualized learning structured upon unique circumstances, user preferences and knowledge background (Mulwa et al., 2012). User preferences in a multimedia learning environment can be supported if users are provided with a possibility to choose the way in which they will perceive information, as it is described in this paper. The question is how to facilitate acquiring computer literacy by making it appealing to one user and at the same time to any other? A possible answer is: by accommodating different perceptual modalities in a virtual learning environment and conducting research on user behaviour.

\section{Multimedia Instructional Design}

Instructional design is a discipline that is founded on scientifically based theoretical principles of learning and instruction (Smith and Ragan, 2005). Accordingly, multimedia instructional design should be grounded on scientifically based theoretical principles of learning through multimedia. In an earlier educational practice, multimedia distance learning indicated the transmittance of educational content remotely via multiple media, such as television, radio and newspapers. Today, computer technology is the main medium in multimedia distance education. Despite the differences, both behavioural and cognitive approaches are used in multimedia design. Both approaches assume analysis, decomposition and simplifying tasks. Both approaches tend to attract the user, and put users' attention into focus, as well as emphasize the importance of the builtin feedback. Furthermore, both approaches highlight meaningful learning in realistic contexts for the application of knowledge and skills (Atkins, 1993).

Mayer (Mayer, 2001; 2005) developed a Cognitive Theory of Multimedia Learning that is based upon Paivio's Dual Coding Theory (Paivio, 1990) and Sweller's Theory of Cognitive Load (Sweller, 1994). The Cognitive Theory of Multimedia Learning is based on the idea that human beings have two separate, but interrelated memory channels for information processing, the visual and the verbal channel. Image processing occurs mainly in the visual channel, and spoken word processing in the verbal. However, the processing of printed words begins in the visual channel, and then moves to the verbal. According to Sweller, the content load should be dosed, taking into account the cognitive processes and limited cognitive capacity. Each of these channels is limited in capacity, while active learning requires coordination of cognitive processes. In multimedia environments, students construct knowledge by choosing words and images from different materials and by combining the verbal and the visual with one another.

There are five cognitive processes in learning through multimedia (Mayer, 2001): 1. Selection of relevant words from displayed text or narration, 2. Selection of relevant images from illustrations, 3 . The organization of selected words 
in a coherent verbal presentation, 4 . The organization of selected images into a coherent visual representation and 5. Integration of the visual and the verbal and the existing knowledge.

Learning through multimedia is learning from words and pictures (Mayer, 2001). Words can be spoken or written and pictures can be static or dynamic. There are five different elements of multimedia: text, images, sound, animation and video. Two of these are static: text and images, and three are dynamic: sound, animation and video (Boyle, 1997). Video can consist of all of the multimedia elements, and there are different approaches in designing a video.

\section{Video}

Video is a powerful tool for conveying information, and can include all elements of multimedia. It requires high-quality software and hardware solutions. The emergence of digital video has enabled users to interact with the displayed content. Interactive video encourages attention and user activity in an educational context. There are several different types of video display among which instructors can choose the appropriate type of video to support e-learning, in accordance with the capabilities and resources that are available (Boyle, 1997).

\section{Video as a text replacement}

Instructors can record a video in addition to the explanations that follow a paragraph of text or lecture presentations. This kind of video makes teaching more interactive and allows users to process the content at their own pace.

\section{Narration}

Instructors can retell the entire contents of a page or screen. This type of video can be tedious and seemingly uninteresting since interactivity is not emphasized. However, the suitability of this technique depends significantly on the educational content.

\section{Scenario}

The scenario is played by actors and recorded to demonstrate certain situations. It is mainly used as a representation of certain activities in various courses.

\section{Simulation}

Users are fully involved in the course of such a video, and it is best suited for interactive online learning. This kind of videos suit learners who prefer to learn in a practical way. Creating a simulation is challenging, but the final product has many advantages. The main feature of the simulation is the interactive environment in which the user's actions affect the future course of events (Boyle, 1997). 


\section{Demonstration}

This kind of video is suitable for detailed guidance on how to do something. Such videos fit well in a teaching process of how to acquire procedural knowledge and skills. Video materials used in the online course from this research are designed as demonstration videos.

\section{Multimodal and adaptive learning}

For the learning process to be effective, it is necessary that the system adapts to individual needs. The design of a LMS tends to go beyond the universal and traditional one-size-fits-all approach (Marshall, 2011). Adaptation of learning is a process in which users are changing the way of learning indirectly, by navigating through the system. In traditional systems, the teacher adapts the teaching contents to the target users. With adaptive learning systems, personalized access to content can rely on the availability of pre-designed resources, and not necessarily on an open corpus of material (Brusilovsky, 2001), which today is a challenge in the field of hypermedia learning or adaptive hypermedia. Although adaptive learning systems are increasingly being developed in the area of TEL, it is still an area where there is a lack of publicly available, comparable, interoperable and reusable data sets covering formal and informal learning (Sosnovsky and Brusilovsky, 2012). Recommender systems are increasingly being used in education. A recommender system, in the context of e-learning, is an agent trying to recommend specific actions based on data gathered from students' previous actions and achievements as well as data on "similar" students. Through educational hypermedia, recommender systems tend to recommend activities to students depending on their goals, interests, previous knowledge and other aspects. Different individualization strategies in conventionally adaptive learning systems support individuals in the process of knowledge acquisition (Mulwa et al., 2012).

Adaptive hypermedia systems use adaptive presentation and support adaptive navigation. A method of adapting various explanations is that the same information is presented via different multimedia forms. In this way, a user is given control over the selection of resources in a multimodal environment. To encourage self-regulated learning, a user is empowered to choose among the resources with regard to personal preferences. At the same time, the diversity of elements in instructional methods could be present in a multimodal environment. Multimodality refers to the multiplicity of methods available for transmitting information. Multimedia means multiplicity of elements in the transmission of multimedia information. Multimodality and multimedia, in the context of multimedia learning, are often used interchangeably. However, it should be stressed that multimodal learning environments allow instructional elements to be presented in more than one sensory mode (visual, aural, written) (Sankey and Gardiner, 2010). Considering that the average user is multi-modal and owns several different preferences in the adoption of information, we support the realization of a 
multi-modal learning environment for learning computer literacy, as described in this paper.

\section{Research and Methodology}

The research was conducted at the Department of Information and Communication Sciences at the Faculty of Humanities and Social Sciences in Zagreb University in the winter term of the Academic Year 2012-2013. The research was conducted within Socio-Humanistic Informatics, an online elective course offered to all graduate students at the Faculty. The skills set within the course covers advanced techniques in using MS Office tools in the context of natural language processing and computer literacy. The students attended the course via Moodle. We researched the patterns of user behaviour in interaction with the LMS by investigating the preferences in selecting various multimedia resources: text, pictures accompanied by text and video resources. Every weekly lesson in the course was supported by three resources containing the same content, but in different form (textual resource, pictorial resource and demonstration video). The study included 98 students; 82 females and 16 males. Teaching materials in the form of multimedia resources were structured in the lesson activity module on Moodle. Data analysis in this paper is based on log file data.

\section{Course Material}

The course content was presented through ten weekly lessons structured as activities. In this research, the content for acquiring the necessary knowledge and skills in computer literacy is presented in three types of resources: text, picture accompanied by text and video.

The multi-modal approach to acquiring computer literacy in this paper is based on a multiple choice of learning resources, different in form but equivalent in meaning. Multimedia in this paper is an integral part of the instructional design of educational resources taking into account that text can be written (including text on the screen) or spoken (including soundtracks), and static images (including graphical representation on the screen) or dynamic display (including video).

The content that is offered through the resources is aimed at mastering the required procedural knowledge and skills.

\section{Learning Resources}

Availability of resources is required across learning contexts and models to enable use for varied purposes. Resource-based learning, particularly in digital environments, offers promise for broad applicability across a variety of contexts to provide access to an expanding global library of digital resources (Sankey and Gardiner, 2010). Resource Based Learning (RBL) is an active learning technique based on preparing the students for retrieval and evaluation of information (Kamer, 2011; Hannafin and Hill, 2008). Reusable Learning Objects 
(RLOs) are entities usable in various teaching situations and they fit well in instructional design that supports collaborative learning (Sylvain et al., 2011). RLOs are a reflection of the growing need for fast creation of re-usable materials in e-learning systems, and they are made of small pieces of learning content. This method of learning goes beyond the high cognitive saturation, by supporting the user in finding information, and not necessarily memorizing information.

It is important to obtain correspondence between the perceptual salience or "noticeability" of a feature and its thematic relevance. In static display, various graphical devices such as arrows, circles and boxes that rely on visual contrast can be used to direct viewers' attention of what is relevant. The same can be used to set the direction of attention within a dynamic display (Lowe and Schnott, 2008).

Taking direction of attention into consideration, within all of the three types of resources, there are specifically marked areas in the form of; bolded keywords in text, marked key points in images, or key targets in the dynamic display, i.e. video. Textual resources are designed as a series of structured and concise instructions displaying text only. Pictorial resources are designed as a combination of text and pictures. The content is structured so that images are combined with text. Video resources are designed as demonstration videos. A sequence of procedures was recorded and aligned with the accompanying narration. Also, there is an option to display the spoken text, beneath the animation, in a line that can be displayed or hidden.

According to the Principle of Redundancy (Mayer, 2001; Sweller, 2005) it is better to learn through animation accompanied by narration, than by animation accompanied by both narration and on screen text. However, due to the simplicity of linear information search, we included the option to display text in video resources. Students were able to choose to display videos with accompanying text without sound, or to display animated actions with accompanying text and sound, or display animated action without accompanying text, with the included sound.

\section{Clustering of Access Logs}

Access logs are a type of log data containing records of information on user access. In other words, every user's activity in the system is recorded in log files. We analysed log files of all the students enrolled in the course in order to learn about the nature of student access towards all three types of resources for each of the ten lessons. The purpose was to determine user behaviour in accessing the resources with the aim of identifying preferences in selecting the resources. Table 1 shows the descriptive statistics of cumulative access towards a resource. 
Table 1. Descriptive statistics of students' access to resources, obtained from $\log$ files

\begin{tabular}{l|c|c|c|c|c|c} 
& Mean & Median & Mode & Min & Max & Range \\
\hline Textual & 9,7 & 6 & 1 & 0 & 48 & 48 \\
Pictorial & 25,7 & 26 & 26 & 0 & 79 & 79 \\
Video & 9,7 & 4 & 1 & 0 & 53 & 53
\end{tabular}

As a data mining approach we used clustering, a method of unsupervised classification of entities displayed as data points or vector features into groups called clusters. Clustering is used in a variety of professional and scientific fields, and is also one of the commonly used methods of data mining in education. It is used for the purpose of grouping users in the so-called clusters according to similarities in behaviour. In e-learning, clustering can be used to find clusters of students with similar characteristics (Romero et al., 2007.). We used the clustering method in order to group the students with similar behaviour patterns into groups. This method was chosen because the students were not limited or directed towards using one type of resource. Instead, the students were able to access all three resources in every lesson, at any time. Therefore, it was possible to group the students according to their access to the resources throughout 10 lessons, each of which contains three different types of resources.

We used a hierarchical clustering method, where it is not necessary to predefine the number of final clusters. Connection type determines the way in which the distance between two clusters is calculated, and thus distinguishes between the following: the distance between two nearest entities (single linkage), the distance between two furthest entities (complete linkage) and the average distance between all entities (average linkage). We chose the complete linkage algorithm due to clearer results.

The process of clustering involves three main steps: displaying entities, calculation of the similarity matrix and the clustering procedure. The entities are most often represented as a set of features that describe the entity. Vectors may contain discrete or continuous values indicating the degree of relevance of features for a particular entity. In this research, the frequencies of students' access to particular type of resources are indicated as values.

Moreover, it is necessary to determine a function that allows the calculation of the similarity matrix expressing similarity between the data points. In this research, the cosine similarity measure is obtained. It is a measure that is identical to the scalar product of two normalized vectors.

The advantage of the cosine similarity is that the measure is outlier-resistant (Ljubešić, 2009). Furthermore, the cosine similarity is identical to Pearson of Spearman correlation coefficient if data is normalized by the $\mathrm{Z}$ value. 


\section{Modelling user activity}

The strategy for modelling user activity in accessing resources (adapted according to the "strategy to modelling student activity in online discussion forums" by Cobo et al. (2011) was conducted as following:

1. Specifying data

a) defining the number of users (98 students),

b) choosing objects for tracking access (30 resources)

c) defining the type of access (resource view)

2. Constructing the series

d) defining the type and the number of samples (30 resources)

e) defining the values from the series' data (the frequency of access)

3. Obtaining the similarity matrix

f) defining the similarity measure (cosine)

g) defining the linkage method (complete linkage)

4. Identifying the obtained clusters which are visualized by a dendrogram (see Figures 1 and 2).

\section{Data analysis and results}

The aim of the research was to establish the existence of user preferences in the use of resources in order to test the consistency of the students choosing a particular type of resource over time. User access to resources is monitored for a time period of 1 semester, tracked through 10 teaching lessons, each containing 3 types of resources, making a total of 30 features. Time continuity of access to resources is given by the following expression:

$$
x^{(i)}=\left(x_{1}^{(i)}, x_{2}^{(i)}, \ldots, x_{n}^{(i)}, \ldots, x_{N}^{(i)}\right)
$$

In the expression, $\mathrm{x}^{(\mathrm{i})}$ marks the $\mathrm{i}^{\text {th }}$ student, $\mathrm{x}_{\mathrm{n}}^{(\mathrm{i})}$ marks the value that $\mathrm{x}^{(\mathrm{i})}$ has for the $\mathrm{n}^{\text {th }}$ resource, and $\mathrm{N}$ is a total number of resources included in the time series $(\mathrm{N}=30)$. Moreover, $\mathrm{x}_{\mathrm{n}}^{(\mathrm{i})}$ is defined the following way where $\mathrm{r}_{\mathrm{n}}^{(\mathrm{i})}$ represents the access frequency of the $\mathrm{i}^{\text {th }}$ student towards the $\mathrm{n}^{\text {th }}$ resource.

$$
x_{n}^{(i)}=r_{n}^{(i)}
$$

The steps in the process of the agglomerative hierarchical clustering, following complete linkage and by calculating the cosine similarity, are the following:

- Vectors are shown quantitatively, expressed as values that represent the frequency of access to a variety of multimedia resources. Those values are obtained by log file analysis. 
- The similarity between entities (a total of 30 ) is calculated using the cosine measure in order to obtain the similarity matrix showing distance between any two data points from a total of 30 data points.

- Agglomerative hierarchical clustering identified three clusters of access to multimedia resources (see Figure 2):

- pictorial cluster

- textual cluster

- video cluster.

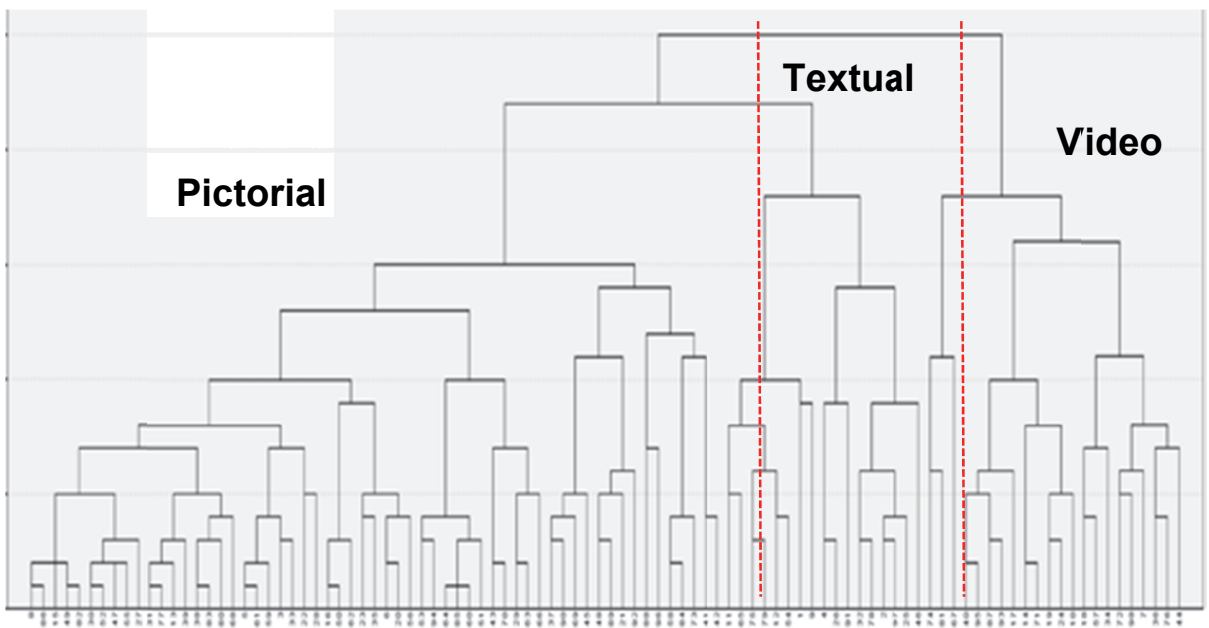

Figure1. Dendrogram shows 3 clusters of students

Cluster analysis shows that the students were consistent in selecting a single type of the resources, due to the fact that three coherent clusters were isolated. According to Figure 1, 60\% of students were selecting pictorial resources, 23\% selected the video resources and $17 \%$ of students selected textual resources. As displayed in Figure 2, the activity of users is grouped into three distinct clusters: text, picture, and video cluster, with a $100 \%$ purity of the clusters. Data mining results obtained by cluster analysis confirm consistent user preferences in selecting multimedia resources.

\section{Discussion}

Multimedia presentation, multimodality, adaptivity and interactivity set different design challenges, so different learning theories such as adaptive learning theory, multimedia learning, as well as learning style theories should be taken into consideration.

Adaptive hypermedia systems use adaptive presentation and support adaptive navigation. Multimodality refers to the multiplicity of methods available for transmitting information. Multimedia includes a combination of media for in- 
formation presentation. While adaptive hypermedia systems support content and presentation adaptivity, in self-regulated learning a user is empowered to choose among the learning resources with regard to personal learning preferences, as described in this paper.

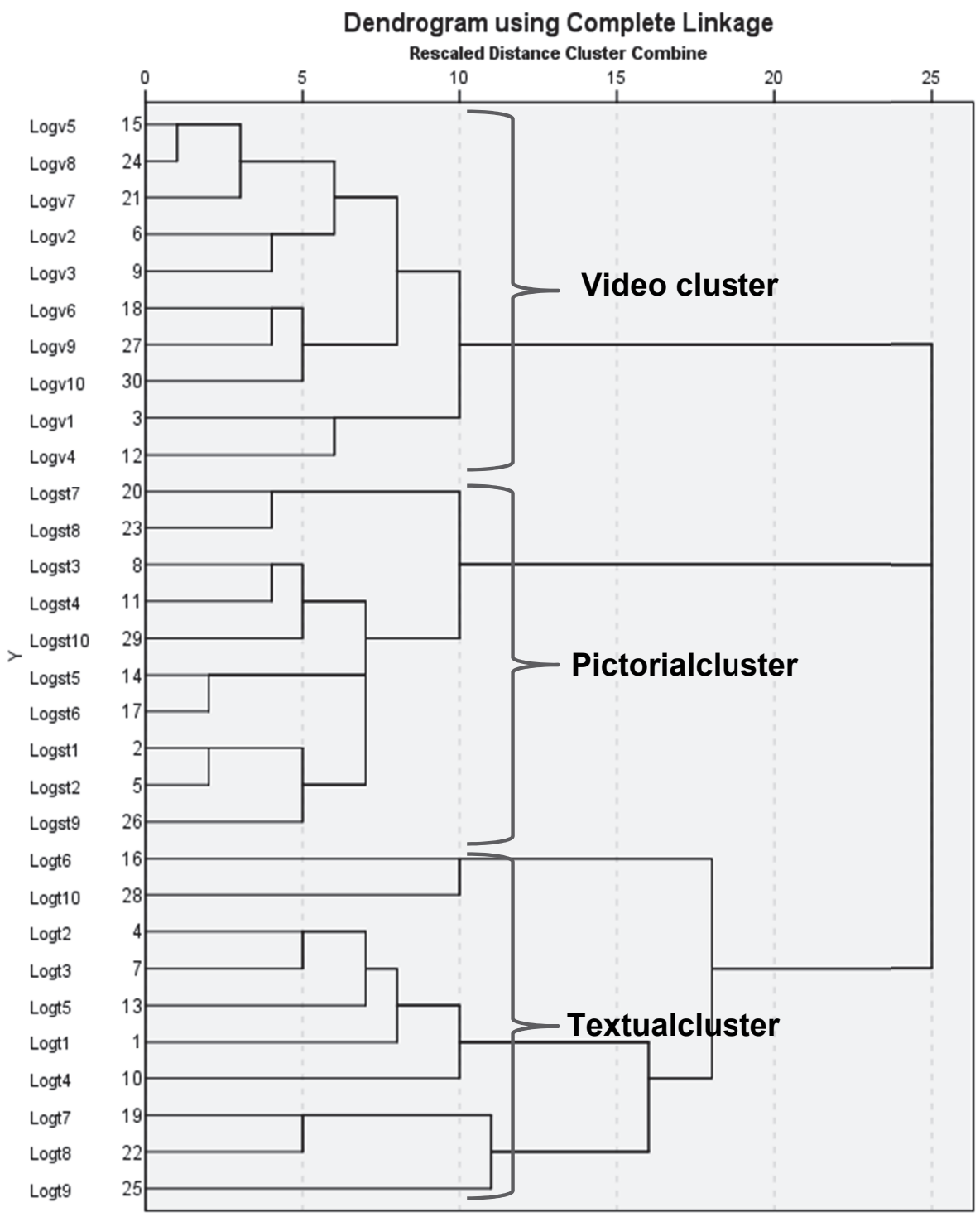

Figure 2. Dendrogram shows 3 coherent clusters of resources, $\log =$ frequency of access; $\mathrm{t}=$ textual, $\mathrm{st}=$ pictorial, $\mathrm{v}=\mathrm{video} ; \mathrm{n}=1,2, \ldots, 10$ - considering 10 lessons 
There are four main categories of learning preferences considering sensory modality. As defined by VARK learning styles model they are described as following: Visual, Aural, Visual, Read/Write, Kinesthetic. Visual learners learn best by seeing the material from visual displays. Aural learners learn best when processing the information by listening. Read/Write learners are those who prefer information displayed as words. Kinesthetic learners prefer examples, labs, demonstrations, simulations, videos, etc.

It is important to take into consideration that the average user is multi-modal and owns several different preferences in the adoption of information. In order to support user to choose among the learning resources with regard to personal learning preferences, we support the realization of a multi-modal learning environment. The vast majority of students agreed that the possibility of selecting a preferred type of resource helped them in acquiring the required knowledge and skills.

Furthermore, we confirmed that the students have consistent preferences in selecting multimedia resources. Also, we found matching results by obtaining feedback responses about users learning preferences in our preliminary research, (Kišičcek et al., 2012.). In our previous work (Lauc et al., 2012a; Lauc et al., 2012b; Lauc et al., 2014) we conducted a research on students' perceptual modes and their learning activity with respect to multimedia learning resources. The feedback results contributed to the fact that user preferences are consistent through time and that students do have their own preferred mode for gaining computer literacy.

This finding should encourage instructors to offer different multimedia resources to their learners in a virtual learning environment, whether the resources are purposefully made or found on the Internet. When teaching know-how skills, efficiency and practicality are the values that matter, hence the user experience is crucial.

Finally, the main goal of multimodal and multimedia learning is to combine different types of media as well as different types of learning resources $n$ order to improve acquiring of knowledge.

\section{Conclusion and Further Research}

Considering multi-modality in the framework of multimedia learning, certain media types can support different learning strategies. Individualization strategies in instruction can affect user experience while learning. In order to foster satisfaction in interaction with the learning material, users should be provided with the possibility to choose the way in which they will perceive information according to their personal preferences. Furthermore, in order to develop their own learning strategies, learners need to have control over their learning process and they need to be empowered in deciding what format of instruction to use. Self-regulation in the learning process can be accomplished through the use of re-usable learning objects, in resource based learning, as described in this paper. 
In this research we described the design of a multimedia e-learning course for gaining computer literacy and investigated student behaviour regarding their access to different types of multimedia resources. By clustering on access log data, as well as obtaining feedback about user learning preferences in preliminary research, we confirmed that students have consistent preferences in selecting multimedia resources throughout the course, meaning that user behaviour patterns could be determined by the regularity in choosing a preferred resource over time.

Interactive video material could be added as an additional type of multimedia resource. In the upcoming research, conducting a study in order to compare the learning behaviour and learning outcomes, should also be taken into consideration.

\section{Acknowledgements}

We would like to thank our project partners from the University Computing Centre in Zagreb for the collaborative work in creating video tutorials within the Project of developing the e-course.

\section{References}

Atkins, M. J. Theories of Learning and Multimedia Applications: An overview. Research Papers in Education, 8(2), 251-271 (1993)

Boyle, T. Design for Multimedia Learning (1st ed.). Prentice Hall Europe (1997)

Brusilovsky, P. Adaptive Hypermedia. User Modelling and User-Adapted Interaction, 11(1-2), 87-110 (2001)

Cobo, G.; García, D.; Santamaría, E.; Morán, J.; Melenchón, J.; Monzo, C. Modeling students' Activity in Online Discussion Forums: a Strategy Based on Time Series and Agglomerative Hierarchical Clustering. In Proceedings of the 4th International Conference on Educational Data Mining (pp. 253-258) (2011)

Hannafin, M. J.,; Hill, J. Resource-based learning. In Handbook of Research on educational communications and technology, pp. 525-536 (2008)

Kammer, J.;Thompson, T. Information-Literate Avatars: Resource-Based Learning in Second life. In Teacjing Information Literacy Online (pp. 109-132). USA: Facet Publishing (2011)

Kišiček, Sanja; Lauc, Tomislava; Golubić, Kruno. Students' Learning Preferences in a Multimedia Online Course. // International journal of education and information technologies. 6 (4), pp: 319-326 (2012)

Lauc, Tomislava; Kišiček, Sanja; Bago, Petra. Multimedia Resources in an Online Course: Access and Usage with Respect to Sensory Modality. // Croatian Journal of Education. 16 (2014), 3; 155-173

Lauc, Tomislava; Kišiček, Sanja; Bago, Petra. Students' Usage and Access to Multimedia Learning Resources in an Online Course with Respect to Individual Learning Styles as Identified by the VARK model//21st Century Learning for 21st Century Skills (LNCS 7563)/ Ravenscroft, Andrew ; Lindstaedt, Stefanie ; Delgado Kloos, Carlos ; Hernández-Leo, Davinia,editor(s). Saarbrücken : Springer, 2012. 548-548

Ljubešić, N. Event detection in parallel information sources (doctoral thesis). The University of Zagreb (2009)

Lowe R., W. Schnot. A Unified View of Learning from Animated and Static Graphics. In Learning with Animation: Research Implications for Design. Ed Lowe R, W. Schnot, pp. 304-357. Cambridge University Press (2008) 
Marshall, K. Choose the Right Video Type for Your Learning Goals. LectoraUniversity (2011)

Mayer, R., E. Multimedia Learning, pp. 41-61, Cambridge University Press (2001)

Mayer, Richard E. Cognitive Theory of Multimedia Learning. In The Cambridge handbook of multimedia learning. Cambridge University Press (2005)

Mulwa, C.; Lawless, S.; O'Keeffe, I.; Sharp, M.; Wade, V. (2012). A recommender framework for the evaluation of end user experience in adaptive technology enhance learning. International Journal of Technology Enhanced Learning, 4(1-2), 67-84 (2012)

Mulwa, C., Lawless, S., O’Keeffe, I., Sharp, M., \&Wade, V. (2012). A Recommender Framework for the Evaluation of End User Experience in Adaptive Technology Enhance Learning. International Journal of Technology Enhanced Learning, 4(1-2), 67-84 (2012)

Nielsen, Jakob. Multimedia and hypertext: the Internet and beyond, Academic Press ; Morgan Kaufmann (1995)

Paivio, A. Mental Representations: Ad Dual Coding Approach. USA: Oxford University Press (1990)

Romero, C.; Ventura, S., García, E. Data Mining in course management systems: Moodlecase study and tutorial. Computers \& Education (2007)

Sankey, M.; Birch, D.; Gardiner, M. Engaging Students Through Multimodal Learning Environments: The journey continues. In Proceedings ASCILITE 2010: 27th Annual Conference of the Australasian Society for Computers in Learning in Tertiary Education: Curriculum, Technology and Transformation for an Unknown Future (pp. 852-863). University of Queensland (2010)

Smith, P. L.; Ragan, T.; J. Instructional Design (3rd ed.). USA: Wiley Publishing (2005)

Sosnovsky, S.; Hsiao, I.-H.; Brusilovsky, P. Adaptation "inthewild": Ontology-Based Personalization of Open-Corpus Learning Material. In21st Century Learning for 21 st Century Skills, Lecture Notes in Computer Science (Vol. LNCS 7563, pp. 425-431). Springer (2012)

Sweller, J. Cognitive Load Theory, Learning Difficulty and Instructional Design. Learning and Instruction, 4, 295-312 (1994)

Sweller, J. The Redundancy Principle in Multimeda Learning. The Cambridge Handbook of Multimedia Learning (Edited by R.E. Mayer.). Cambridge University Press (2005)

Sylvain, M. C.; Mofford, K.; Lehr, E.; Riley, J. E. Reusable learning objects: Developing Online Information Literacy Instruction through Collaborative Design. In Teaching Information Literacy Online (pp. 25-43). Facet Publishing (2011) 